\title{
Effect of surface interactions on the hysteresis of capillary condensation in nanopores
}

\author{
F. Casanova ${ }^{1(a)}$, C. E. Chiang ${ }^{1}$, C.-P. Li ${ }^{1}$, I. V. Roshchin ${ }^{1,2}$, A. M. Ruminski ${ }^{3}$, M. J. Sailor ${ }^{3}$ and \\ I. K. SCHULlER ${ }^{1}$ \\ ${ }^{1}$ Physics Department, University of California - San Diego, La Jolla, CA 92093, USA \\ 2 Physics Department, Texas A\&M University - College Station, TX 77843, USA \\ ${ }^{3}$ Department of Chemistry and Biochemistry, University of California - San Diego, La Jolla, CA 92093, USA
}

received 18 September 2007; accepted in final form 12 November 2007 published online 7 December 2007

PACS 64.70.Fx - Liquid-vapor transitions

PACS 81.07.-b - Nanoscale materials and structures: fabrication and characterization PACS $05.70 . \mathrm{Np}$ - Interface and surface thermodynamics

\begin{abstract}
Gas adsorption and liquid desorption of a number of organic vapors in anodized nanoporous alumina, with controlled geometry (cylindrical pore diameters from 10 to $60 \mathrm{~nm}$ ), are studied using optical interferometry. The narrow-diameter distribution of disconnected pores allows checking the validity of the (long-predicted but not experimentally verified) Kelvin equation without any adjustable parameters, modeling or other assumptions. Evaporation occurs at liquidvapor equilibrium according to this equation, whereas condensation occurs from metastable states of the vapor phase by nucleation, enhanced by surface defects inside the nanopores. This produces hysteresis, in qualitative agreement with theoretical models and simulations that use Van der Waals interactions between the fluid and the pore surface. The reproducibility of the hysteresis depends on the strength of these interactions, which play an important role in the dynamics of capillary condensation.
\end{abstract}

Copyright (c) EPLA, 2008

Introduction. - Phase transitions in confined geometries are being intensely studied recently because materials can be prepared in which the relevant sizes are comparable to characteristic physical sizes which control the transition mechanisms. In particular, capillary condensation can be studied in nanoporous materials in which the pore sizes are only somewhat larger than interatomic spacing (and are comparable to the inverse of the curvature of the meniscus of liquids of interest). In these cases, phase transitions in systems such as simple fluids [1] or polymers [2] may be affected by confinement phenomena, curvature of surfaces, roughness, interactions with the surfaces, etc. Porous materials have been crucial for studies of superfluidity [3], capillary condensation in disordered-interconnected $[4]$ and self-assembled pores in a variety of configurations $[5,6]$. These materials are important for applications such as bio- [7] and chemical sensing [8] and as nanotemplates [9]. Capillary condensation studies in confined geometries are also relevant for research of thermocapillarity [10], motion of fluids

${ }^{(a)}$ E-mail: casanova@physics.ucsd.edu in constrained geometries [11] or avalanche dynamics of first-order phase transitions [12]. In spite of this, comparisons with theoretical models are hindered by the lack of studies and/or availability of porous materials with well-controlled and narrow pore size distribution (PSD), in particular for pore diameters $\geqslant 10 \mathrm{~nm}$.

In this letter, we present a study of capillary condensation of organic vapors in nanoporous alumina with cylindrical pores open at one end and well-controlled sizes in the 10 -to- $60 \mathrm{~nm}$ diameter range. We have successfully used very sensitive optical interferometry $[7,8]$ to obtain adsorption-desorption isotherms. We observe capillary evaporation of the condensed vapor from the nanopores at relative pressures which are in excellent quantitative agreement with the prediction of the classical Kelvin equation [13] without any adjustable parameters, modeling or other assumptions (i.e., all parameters are determined experimentally). This is the first time that the validity of the Kelvin equation has been checked in independent regular pores for diameters $\geqslant 10 \mathrm{~nm}$. On the other hand, capillary condensation occurs from metastable vapor states, giving rise to hysteresis, in qualitative agreement 


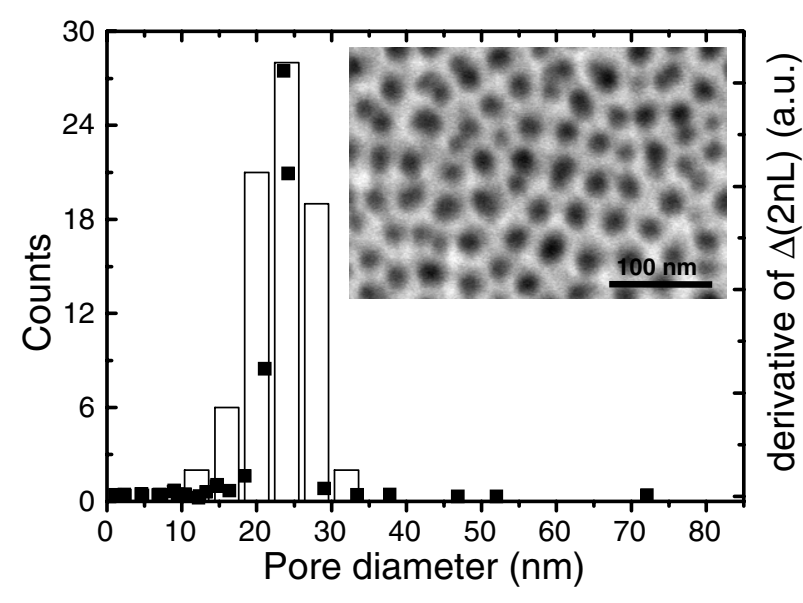

Fig. 1: Pore size distribution for the $22 \mathrm{~nm}$ pore sample obtained from SEM images (histogram, left axis) and from the derivative of the desorption isotherm for isopropanol combined with the Kelvin equation (solid squares, right axis). Inset: planview SEM image of the $22 \mathrm{~nm}$ pore sample.

with theoretical and simulation studies that use Van der Waals interactions at the fluid-solid interface [14-19]. Moreover, we also show that the reproducibility of the hysteresis depends on the strength of these interaction forces. This is important for the development of a theory of the poorly understood dynamics of the hysteresis (see, for example, ref. [4]), a phenomenon known for over a century.

Experimental. - Nanoporous alumina samples were prepared by e-beam evaporation of 6 - $\mu$ m-thick $\mathrm{Al}$ layers on n-type Si followed by two-step anodization [9,20]. The periodicity and diameters of the pores were controlled by the electrolyte (sulfuric or oxalic acid) and anodization voltage $(10-40 \mathrm{~V})$. Subsequent acid etching for different times was used to widen the pores, without affecting the periodicity and the thickness. Fabrication details are described in ref. [9]. Diameters from 10 to $60 \mathrm{~nm}$ with standard deviations $<20 \%$ were obtained in this fashion. The PSDs were determined from scanning electron microscopy (SEM) images (operating in field emission mode, see fig. 1). The porosities $\rho$ (fraction of empty volume) were obtained from the same images, yielding values from $\sim 0.1$ to 0.5 . Cross-sectional SEM images reveal that the pores are cylindrical, open at one end and disconnected. The thicknesses $(L)$ of the porous layers were measured with profilometry, yielding all similar values $(\sim 6.2 \mu \mathrm{m})$.

Reflectivity spectra were obtained using a fiber-optic/ lens assembly, as previously described [7]. Briefly, white light from a tungsten source is introduced to one arm of the bifurcated fiber and focused onto the sample to a spot size of $1-2 \mathrm{~mm}^{2}$. The reflected light is collected and passed via the other arm to a $350-1000 \mathrm{~nm}$ CCD spectrometer. The incident and reflected light beams are normal to the surface. The spectra show a series of interference fringes

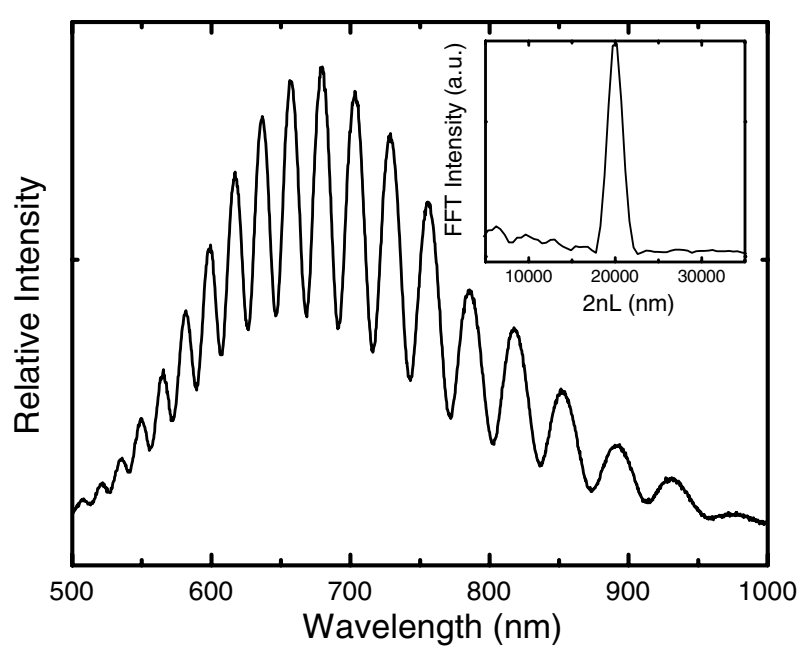

Fig. 2: Reflectance spectrum of the porous alumina layer with a pore diameter of $13 \pm 3 \mathrm{~nm}$. Inset: fast Fourier transform (FFT) of the spectrum, showing the peak corresponding to the effective optical thickness $(2 n L)$ of the layer.

(fig. 2) arising from the reflection of light at the top and bottom interfaces of the porous alumina layer. Since the pore diameter is much smaller than the light wavelength $\lambda$, the porous layer acts as a single medium with an average refractive index, $n$. The fringe maxima are described by the Fabry-Pérot relationship $m \lambda=2 n L$, where $m$ is an integer. The term $2 n L$ is the effective optical thickness of the porous layer. Filling the pores with an analyte results in a change of $n$, shifting the Fabry-Pérot fringes. The value of $2 n L$ can be obtained as the position of the peak in the fast Fourier transform of the reflectance spectrum (inset in fig. 2) [7]. We fit the peak to a Gaussian function in order to determine its center. This optical technique can resolve down to $1 \mathrm{~nm}$ in $2 n L$, which, for typical pore sizes and analytes used in this work, corresponds to a precision of $\sim 2 \cdot 10^{-4}$ in the pore filling fraction.

Gas dosing was performed in a teflon cell fitted with a glass window. Saturated vapor analyte was generated by bubbling pure $\mathrm{N}_{2}$ through the liquid analyte at $25^{\circ} \mathrm{C}$ and then mixing the effluent with pure $\mathrm{N}_{2}$ using a computer-controlled gas dosing system. Total gas flow was kept constant at 500 standard cubic centimeters per minute (sccm). Relative vapor pressures, $P / P_{s}$, where $P_{s}$ is the saturation vapor pressure, were calculated using the published standard vapor pressures of the pure liquids and the dilution ratio of the gas dosing system.

All samples were dosed with isopropanol and toluene vapors. The value of $2 n L$ was monitored while $P / P_{s}$ was increased and subsequently decreased stepwise. Following every $P / P_{s}$ change, the system was allowed to reach an equilibrium, in order to exclude transitional processes from consideration. For each step, the change in $2 n L$ $(\Delta(2 n L))$ with respect to the baseline obtained in pure $\mathrm{N}_{2}$, i.e., with empty pores, was plotted as a function of $P / P_{s}$. Since $\Delta(2 n L)$ is uniquely related to the volume of 
analyte adsorbed into the pores, this provides directly the adsorption-desorption isotherms [13]. Every isotherm was repeated 3-6 times to check reproducibility.

Results and discussion. - All samples exhibit isotherms with similar shape and display hysteresis (see fig. 3 for two different samples and two different analytes). According to the IUPAC classification [21], the shape corresponds to a type-IV isotherm and the hysteretic behavior is type H1. Type-IV isotherms are present in materials with pore diameters of $\sim 2-50 \mathrm{~nm}$. The initial part of the curve (low $P / P_{s}$ ) is attributed to monolayer-multilayer adsorption on the internal surfaces of the pores. The steep increase in slope associated with capillary condensation within the nanopores is followed by saturation at high $P / P_{s}$ as the pores become completely filled with liquid $[13,21]$. The sharpness of the transition in the present case is attributed to the narrow PSD (see fig. 1). The relative pressure at which the transition occurs depends on the pore size and the analyte (see fig. 3). Type-H1 hysteresis has been considered a signature of materials with disconnected cylindrical pores open at both ends [22]. Indeed, the samples in this study have disconnected pores, but are only open at one end. The latter disagrees with the classical Cohan model, which predicts no hysteresis for cylindrical pores open at one end $[13,23]$. This failure was previously mentioned [24] and explained [5] in porous silicon.

To confirm that the saturated values of the adsorption isotherms correspond to the pores completely filled with liquid, $2 n L$ was measured before (empty pores) and after immersion of the samples in liquid analytes. The resulting $\Delta(2 n L)$ values, horizontal lines in fig. 3 , are in agreement with the saturated values of the isotherms. We can obtain $L$ and $\rho$ for each sample by using these two measured $2 n L$ values (for empty and liquid-filled pores) and the Bruggeman effective medium approximation $[7,25]$ :

$$
(1-\rho)\left(\frac{n_{\mathrm{AlO}_{x}}^{2}-n^{2}}{n_{\mathrm{AlO}_{x}}^{2}+2 n^{2}}\right)+\rho\left(\frac{n_{p}^{2}-n^{2}}{n_{p}^{2}+2 n^{2}}\right)=0,
$$

where $n_{\mathrm{AlO}_{x}}=1.65$ is the refractive index of anodized alumina [26] and $n_{p}$ is either 1 for empty pores or the refractive index of the corresponding liquid for liquid-filled pores. $L$ and $\rho$ values are consistent for various liquids (isopropanol, toluene, ethanol, acetone, water, hexane) and with previous characterization measurements.

Since the pore sizes and their dispersion are small, we first analyze the desorption curve because this is expected to be independent of uncontrolled details of the pore surface such as roughness, slight variations in shape, possible contaminants, etc. $[5,14,15]$. Indeed, desorption curves in fig. 3a) show good reproducibility for all analytes. To describe the capillary behavior, we use a macroscopic thermodynamic approach. Historically, the Kelvin equation has been the most frequently used

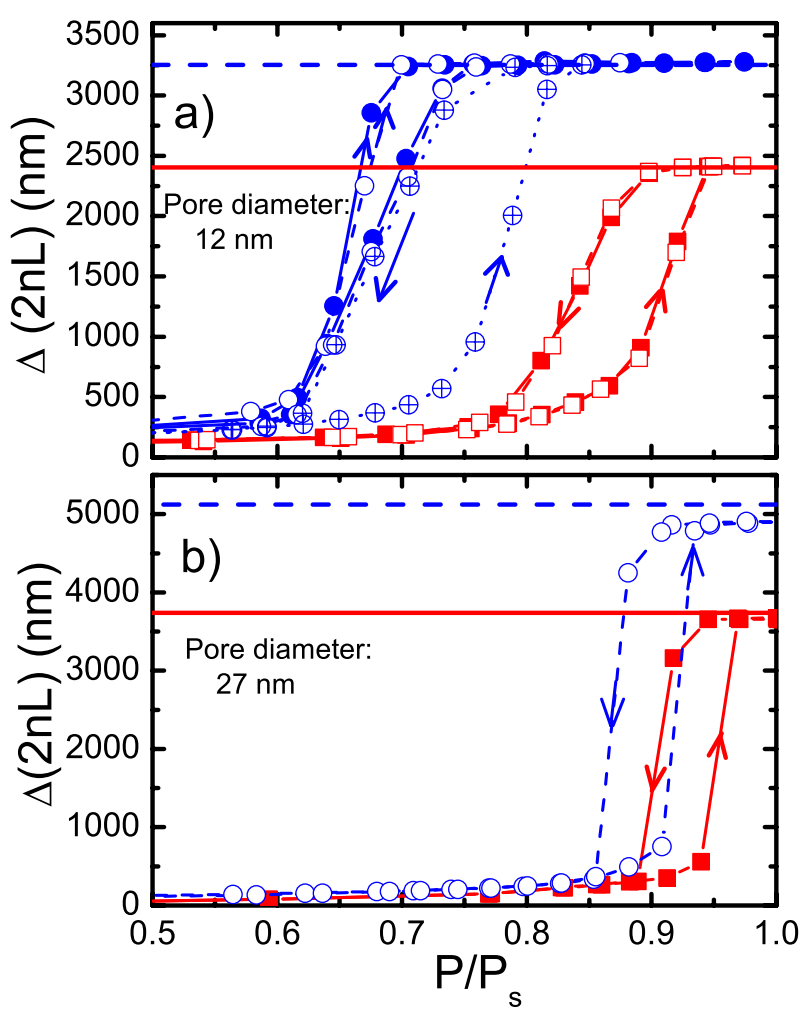

Fig. 3: Change in $2 n L$ as a function of the relative pressure of analyte (squares for isopropanol and circles for toluene). Isotherms were obtained by first increasing (adsorption) and then decreasing (desorption) the relative pressure in discrete steps. The size of the error bars is smaller than the size of the symbols. Three different runs for toluene (different square symbols) and two different runs for isopropanol (different circle symbols) are plotted in (a) to show the degree of reproducibility. Pore diameters of the samples are $12 \pm 2 \mathrm{~nm}$ (a) and $27 \pm 3 \mathrm{~nm}$ (b). Horizontal lines correspond to $\Delta(2 n L)$ measured by using the analytes in liquid phase (solid line for isopropanol, dashed line for toluene).

model [13]. For cylindrical pores with a hemispherical meniscus, the Kelvin equation takes the form

$$
\ln \left(\frac{P}{P_{s}}\right)=-\frac{2 \gamma V_{L}}{R T r_{m}}
$$

where $r_{m}$ is the radius of the liquid meniscus, $R$ is the ideal-gas constant, and $V_{L}$ and $\gamma$ are the molar volume and surface tension of the liquid at temperature $T$. The Kelvin equation is supposed to be valid for pore diameters $\geqslant 7.5-10 \mathrm{~nm}$, according to theoretical approaches [15,27]. However, experimental validation of the Kelvin equation in an ideal geometry has been limited to gas condensed in a slit-like space between mica surfaces [28] and to nanopores $\leqslant 10 \mathrm{~nm}$ in diameter in molecular sieves [29]. In the first case, the adsorbed liquid has a different dimensionality (2D) than a cylindrical pore (1D), in which surface interactions or curvature can be very different and may play an important role. In the second case, silicate MCM-41 and related porous materials have been used to 


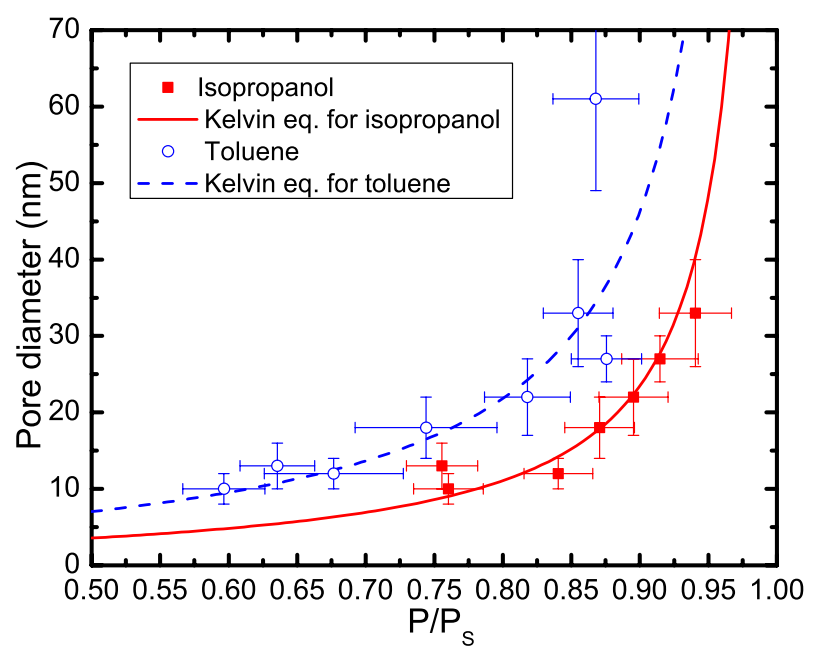

Fig. 4: Pore diameter obtained from SEM as a function of the relative pressure of the analyte (solid squares for isopropanol, open circles for toluene) at which capillary evaporation takes place. The value is taken as the inflection point of the desorption branch. Solid and dashed lines correspond to the Kelvin equation for isopropanol and toluene, respectively.

investigate the validity of the Kelvin equation in regular cylindrical pores with diameters $\leqslant 10 \mathrm{~nm}$. However, in this case, contrary to our direct SEM measurements, the pore structure is obtained from modeling of X-ray diffraction data. Moreover, assumptions must be made regarding the non-negligible thickness $(t)$ of the adsorbed film. In the present case, we can simply use the pore radius for $r_{m}$, because $t$ of the film formed on the pore walls prior to condensation is negligible above $10 \mathrm{~nm}$ pore diameter ${ }^{1}$. Figure 4 shows the relationship between the pore diameters of 8 samples (obtained from SEM) and the relative pressure at which evaporation from the pores occurs on desorption for isopropanol and toluene. The analytical Kelvin equation (dashed and solid lines in fig. 4), using standard values of $V_{L}$ and $\gamma$ for each analyte, is in excellent agreement with the experimental measurement. It is important to stress that this agreement is obtained without any fitting parameters, modeling of the pore structure or the adsorbed film, or other considerations. This agreement also indicates that the pore diameter at the surface of the layer is constant along the pores, confirming the nearly ideal cylindrical shape of anodized alumina nanopores. The width of the transition is caused by the PSD within a sample. Therefore, the PSD of a porous sample can be obtained from the derivative of its desorption isotherm within the transition region. The conversion from relative pressure to pore diameter is obtained by applying the Kelvin

\footnotetext{
${ }^{1} t$ of this adsorbed film as a function of $P / P_{s}$ can be calculated from $\Delta(2 n L)$ values of the adsorption isotherm (which are proportional to the filling fraction of the pores) in the region prior to the capillary condensation, by using a simple geometrical model (see ref. [24]). For the present samples, dosed with isopropanol and toluene, $t$ is negligible compared to the pore diameters used.
}

equation to the experimentally determined desorption curve. The PSDs for our samples are in agreement with the distributions obtained from SEM images (see fig. 1 for an example), thus confirming the validity of the Kelvin equation for capillary evaporation.

The adsorption curve is critically dependent on the adsorbed fluid, i.e., it is highly reproducible for isopropanol whereas significant scatter is observed for toluene. In some cases, when using toluene, the relative pressure for condensation may even occur at a pressure lower than that for evaporation. The results described above are observed in all samples, but shown only for one (fig. 3a)) for the sake of clarity. These can be understood if we take into account the metastable states that can occur in this type of experiment. Calculations using density functional theory $[14,15,17]$, gas lattice models [18], Monte Carlo simulations $[15,16]$ and molecular simulations [19], all incorporating Van der Waals interactions, show that, for disconnected pores, metastability should exist beyond the equilibrium transition given by the Kelvin equation both for adsorption (metastable vapor states) and desorption (metastable liquid states). However, it seems that metastable liquid states are irrelevant on desorption because the preexisting meniscus (liquid-vapor interface) can retreat on pore emptying. Therefore, desorption occurs at the true equilibrium transition, as found experimentally [5,14-17]. On the other hand, on adsorption, the vapor condenses by nucleation in metastable states $[5,14,15]$, giving rise to hysteresis. For cylindrical pores, the origin of this nucleation is attributed to small variations within the pore diameter and in the fluid-solid interactions. The unavoidable disorder present in real samples tends to favor metastability during adsorption by providing nucleation sites for the liquid [5]. This in turn would produce the hysteresis behavior observed. The dynamics of the system in the metastable region has been shown to be activated [4].

Our results clearly show the relevance of fluid-solid interactions to the observed hysteresis. These interactions influence the structure of the adsorbed layer at low relative pressures and, in the present experiments, are thought to be responsible for the significant scatter in the adsorption isotherms for toluene. We suggest that interactions of a polar molecule such as isopropanol [30] with the hydrophilic surface of alumina [30] stabilize the adsorbed layer [13]. In this case, condensation occurs close to the limit of metastability on adsorption and therefore is reproducible. On the other hand, toluene is a nearly nonpolar molecule [30]. A weaker interaction with the pore walls would make the metastable states more sensitive to slight fluctuations (lower energy barriers), resulting in more stochastic condensation behavior. This view is in agreement with an activated dynamics of the condensation [4]. Moreover, the larger surface tension of toluene [30] also favors the formation of liquid seeds, which are very sensitive to initial conditions and surface defects. Such seeds are necessary in the nucleation process for 
condensation, as observed in the porous silicate MCM41 [16]. Further investigation is required to clarify this point.

Conclusions. - In conclusion, controlled pore sizes with narrow distributions make anodized nanoporous alumina an excellent system for studies of gas adsorption and capillary condensation as a means to systematically test theoretical models of capillary condensation. We observe hysteretic capillary condensation of the condensable organic analytes toluene and isopropanol for a wide range of pore diameters (10 to $60 \mathrm{~nm}$ ) using very precise optical interferometry. Capillary evaporation occurs at the equilibrium pressure for all pore sizes and analytes as predicted by the Kelvin equation without fitting parameters, thus validating this equation for regular cylindrical pores in the $10-60 \mathrm{~nm}$ range. On the other hand, capillary condensation occurs through nucleation, and there exists a pressure range of metastability of the gas phase. Such hysteresis depends on the fluid-solid interactions, as shown experimentally. Polar interactions and/or surface tensions of the analytes could explain this dependence, taking into account the activated dynamics of the condensation.

The financial support of the Air Force Office of Scientific Research is gratefully acknowledged. FC acknowledges financial support from Spanish MEC and Fulbright Commission. AMR thanks the University of California, San Diego for a Graduate Assistance in Areas of National Need (GAANN) fellowship.

\section{REFERENCES}

[1] Gelb L. D., Gubbins K. E., Radhakrishnan R. and Sliwinska-Bartkowiak M., Rep. Prog. Phys., 62 (1999) 1573.

[2] Zhang M., Dobriyal P., Chen J.-T., Russell T. P., Olmo J. and Merry A., Nano Lett., 6 (2006) 1075.

[3] Glyde H. R., Plantevin O., FÅK B., Coddens G., Danielson P. S. and Schober H., Phys. Rev. Lett., 84 (2000) 2646.

[4] Valiullin R., Naumov S., Galvosas P., Kärger J., Woo H.-J., Porcheron F. and Monson P. A., Nature, 443 (2006) 965.

[5] Wallacher D., Künzner N., Kovalev D., Knorr N. and Knorr K., Phys. Rev. Lett., 92 (2004) 195704.

[6] Alvine K. J., Shpyrko O. G., Pershan P. S., Shin K. and Russell T. P., Phys. Rev. Lett., 97 (2006) 175503.
[7] Pacholski C., Sartor M., Sailor M. J., Cunin F. and Miskelly G. M., J. Am. Chem. Soc., 127 (2005) 11636.

[8] Gao J., Gao T., Li Y. Y. and SAilor M. J., Langmuir, 18 (2002) 2229.

[9] Li C.-P., Roshchin I. V., Batlle X., Viret M., Ott F. and Schuller I. K., J. Appl. Phys., 100 (2006) 074318 .

[10] Röntzsch L., Heinig K.-H., Schuller J. A. and Brongersma M. L., Appl. Phys. Lett., 90 (2007) 044105.

[11] Moseler M. and Landman U., Science, 289 (2007) 1165.

[12] Lilly M. P., Finley P. T. and Hallock R. B., Phys. Rev. Lett., 71 (1993) 4186.

[13] Gregg S. J. and Sing K. S. W., Adsorption, Surface Area and Porosity (Academic Press, London) 1982.

[14] Ravikovitch P. I., O'Domhnaill S. C., Neimark A. V., Schüth F. and Unger K. K., Langmuir, 11 (1995) 4765.

[15] Neimark A. V., Ravikovitch P. I. and Vishnyakov A., Phys. Rev. E, 62 (2000) R1493.

[16] Ravikovitch P. I., Vishnyakov A., Neimark A. V., Ribeiro Carrott M. M. L., Russo P. A. and Carrott P. J., Langmuir, 22 (2006) 513.

[17] Ball P. C. and Evans R., Langmuir, 5 (1989) 714.

[18] Marconi U. M. B. and Van Swol F., Phys. Rev. A, 39 (1989) 4109.

[19] Heffelfinger G. S., Van Swol F. and Gubbins K. E., J. Chem. Phys., 89 (1988) 5202.

[20] Masuda H. and Fukuda K., Science, 268 (1995) 1466.

[21] Sing K. S. W., Everett D. H., Haul R. A. W., Moscou L., Pierotti R. A., Rouquérol J. and Siemieniewska T., Pure Appl. Chem., 57 (1985) 603.

[22] Grosman A. and Ortega C., Langmuir, 21 (2005) 10515.

[23] Cohan L. H., J. Am. Chem. Soc., 60 (1938) 433.

[24] Coasne B., Grosman A., Ortega C. and Simon M., Phys. Rev. Lett., 88 (2002) 256102.

[25] Bruggeman D. A. G., Ann. Phys. (Leipzig), 24 (1935) 636.

[26] Xu W. L., Chen H., Zheng M. J., Ding G. Q. and Shen W. Z., Opt. Mater., 28 (2006) 1160.

[27] Kruk M., Jaroniec M. and Sayari A., Langmuir, 13 (1997) 6267.

[28] Fisher L. R. and Israelachvili J. N., Nature, 277 (1979) 548.

[29] Morishige K. and TAteishi M., Langmuir, 22 (2006) 4165 and references therein.

[30] Redón R., Vázquez-Olmos A., Mata-Zamora M. E., Ordóñez-MEdrano A., Rivera-Torres F. and Saniger J. M., J. Colloid Interface Sci., 287 (2005) 664 . 